\title{
Real-time prediction and ponding process early warning method at urban flood points based on different deep learning methods
}

\section{Yihong Zhou}

Zhengzhou University

\section{Zening Wu}

Zhengzhou University

Mengmeng Jiang

Zhengzhou University

Hongshi Xu

Zhengzhou University

Denghua Yan

Zhengzhou University

Huiliang Wang ( $\nabla$ wanghuiliang@zzu.edu.cn )

Zhengzhou University https://orcid.org/0000-0003-0057-455X

Chentao He

Zhengzhou University

\section{Xiangyang Zhang}

Zhengzhou University

\section{Research Article}

Keywords: Urban flood, Ponding point, Deep learning, Prediction, Early warning

Posted Date: January 24th, 2022

DOI: https://doi.org/10.21203/rs.3.rs-1114192/v1

License: (c) (1) This work is licensed under a Creative Commons Attribution 4.0 International License. Read Full License 


\title{
Real-time prediction and ponding process early warning method at urban flood points based on different deep learning methods
}

\author{
Yihong ZHOU a , Zening WU a, Mengmeng JIANG ${ }^{\text {a }}$, Hongshi XU a, Denghua YAN ${ }^{\text {a }}$, Huiliang WANG a, *,
}

Chentao $\mathrm{HE}^{\text {a }}$, Xiangyang ZHANG ${ }^{\mathrm{a}}$

${ }^{a}$ College of Water Conservancy Engineering, Zhengzhou University, Zhengzhou, Henan, 450001, P. R. China

Abstract: Accurate prediction of urban floods is regarded as one of the critical means to prevent urban floods and reduce the losses caused by floods. However, due to the uncertainty and complexity of urban floods and waterlogging, it is very difficult to use simulation models to quickly and accurately predict and warn of urban floods. Therefore, it is necessary to develop new methods to support the rapid and accurate prediction of urban floods and waterlogging. In this study, a refined prediction and early warning method for urban flood and waterlogging processes based on deep learning methods is proposed from three aspects: research feasibility, method applicability, and method system. The spatial autocorrelation of rain and ponding points is analyzed by Moran's I. For each ponding point, the relationship model between the rainfall process and ponding process is constructed based on different deep learning methods, and the results are analyzed and verified by a statistical evaluation method. The results show that the gradient boosting decision tree algorithm has the highest accuracy and efficiency (with a root mean square error of 0.001 ) for ponding process prediction and is regarded as the most suitable method for ponding process prediction. Finally, the real-time prediction and early warning of urban floods and waterlogging processes driven by rainfall forecast data are realized, and the

\footnotetext{
*Corresponding author at: College of Water Conservancy Engineering, Zhengzhou University, Zhengzhou, Henan, 450001, P. R. China

Tel: +8618135671702

E-mail address: wanghuiliang@126.com
} 

results are verified by the measured data. In addition, the results of the sensitivity analysis show that the rainfall peak and location coefficient have the greatest impact on ponding.

Key words: Urban flood; Ponding point; Deep learning; Prediction; Early warning 


\section{Introduction}

In recent years, under the background of global warming and urbanization (Masson et al., 2020;

Kenney and Janetos, 2020), urban flooding and waterlogging events have occurred frequently worldwide and have become a serious challenge faced by many large cities worldwide (Moghadas et al., 2019; Wang et al., 2020; Thanvisitthpon et al., 2020; Sharifi, 2020). For example, in July 2019, heavy rains caused at least 18 deaths in Mumbai, India, and caused largescale traffic interruptions (Ke et al., 2020). In July 2021, the "7.20 rainstorms" in Zhengzhou, China, caused 292 deaths and a direct economic loss of 53.2 billion yuan. With the aggravation of climate change, the frequency and intensity of extreme rainfall events may increase in the future, and cities may face a greater flood disaster threat (Schreider et al., 2000; Ntelekos et al., 2010; Xu et al., 2018). Therefore, in the urban flooding and waterlogging process, how to forecast the inundation process in a more accurate way for each ponding point to minimize the life, property and economic losses caused by floods has become a scientific problem to be solved urgently.

To prevent the occurrence of floods and implement timely countermeasures to reduce flood losses, city authorities usually need to predict urban flooding and waterlogging (Ke et al., 2020). Urban flood simulation models based on physical mechanisms are the most popular method for predicting the urban flooding process and have attracted great attention from scientists (Mignot et al., 2018). Many scholars have discussed urban floods and waterlogging simulations from different angles (Babaei et al., 2018; Bermudez et al., 2018; Hou et al., 2020). However, due to the lack of calibration and verification data (Schmitt et al., 2004) and the long running time of the model (Suwit and Parinda., 2016; Hou et al., 2020), these physical models are limited to a 
certain extent in the real-time urban flood prediction and early warning. In contrast, the deep learning technology (or machine learning) can mine knowledge and laws that conventional data processing methods cannot mine and identify highly complex nonlinear relationships between characteristic and predictive variables (Panahi et al., 2020), and it does not need to understand the potential physical process (Mosavi et al., 2019). Therefore, deep learning technology provides a new idea for solving the problem of rapid and accurate urban floods prediction, which is particularly useful in large- and medium-sized cities with sufficient data. In recent years, many deep learning methods have been used in urban flood research. Several types of deep learning methods, gradient boosting decision trees (GBDTs) (Wu et al., 2020; Rahebeh et al. 2020), support vector machines (SVMs) (Nayak and Ghosh, 2013; Wang and Song, 2019) and neural networks (Singh et al., 2010; Lei et al., 2021), have been successfully applied to urban flood prediction. However, in urban flood forecasting research, not all in-depth learning methods are suitable for urban flood research, and the prediction performance of each method may vary greatly due to algorithms and specific prediction requirements. Therefore, it is necessary to develop a relatively complete set of methods using deep learning methods to predict the urban flood ponding process to improve the urban flood prediction method and guide urban flood control.

Based on the aforementioned literature, this study analyzed the feasibility of the research method, compared the applicability of different deep learning technologies in urban flood prediction, and applied a deep learning method to propose a set method system of urban flood ponding process prediction. The specific objectives of the study were to i) analyze the feasibility of the urban flood ponding process prediction for each ponding point based on the spatial 
autocorrelation method, ii) use GBDT, SVM and backpropagation neural network (BPNN) to construct the relationship model between the rainfall process and the ponding process and use statistical evaluation methods to analyze the accuracy of different deep learning methods, which aims to propose a method suitable for predicting the urban flood ponding process, and iii) construct the urban flood ponding process prediction and early warning method driven by rainfall forecast data, combined with measured data to test the accuracy of the early warning results. The novelty of this work lies in proposing a rapid and accurate prediction method system for the urban flood ponding process based on the method feasibility, applicability of the method, and technical system. The research results can provide technical references for urban flood early warning and urban flood control.

\section{Study area and data processing}

\subsection{Study area}

Zhengzhou is the capital city of Henan Province and an important transportation hub in central China. As shown in Fig. 1. It is located in northern central Henan Province, with high terrain in the west and low terrain in the east. It has a temperate continental monsoon climate, with an average annual precipitation of $639.5 \mathrm{~mm}$. However, the precipitation distribution in Zhengzhou is very uneven. The precipitation in the flood season (June to September) accounts for approximately $60 \%$ of the annual precipitation. In response to urban floods and waterlogging, Zhengzhou City has taken various measures in recent years, such as dredging rivers and cleaning up sewers. However, the flooding and waterlogging phenomenon still occurs frequently, which seriously threatens the safety of people's lives and property and the normal operation of the city. 


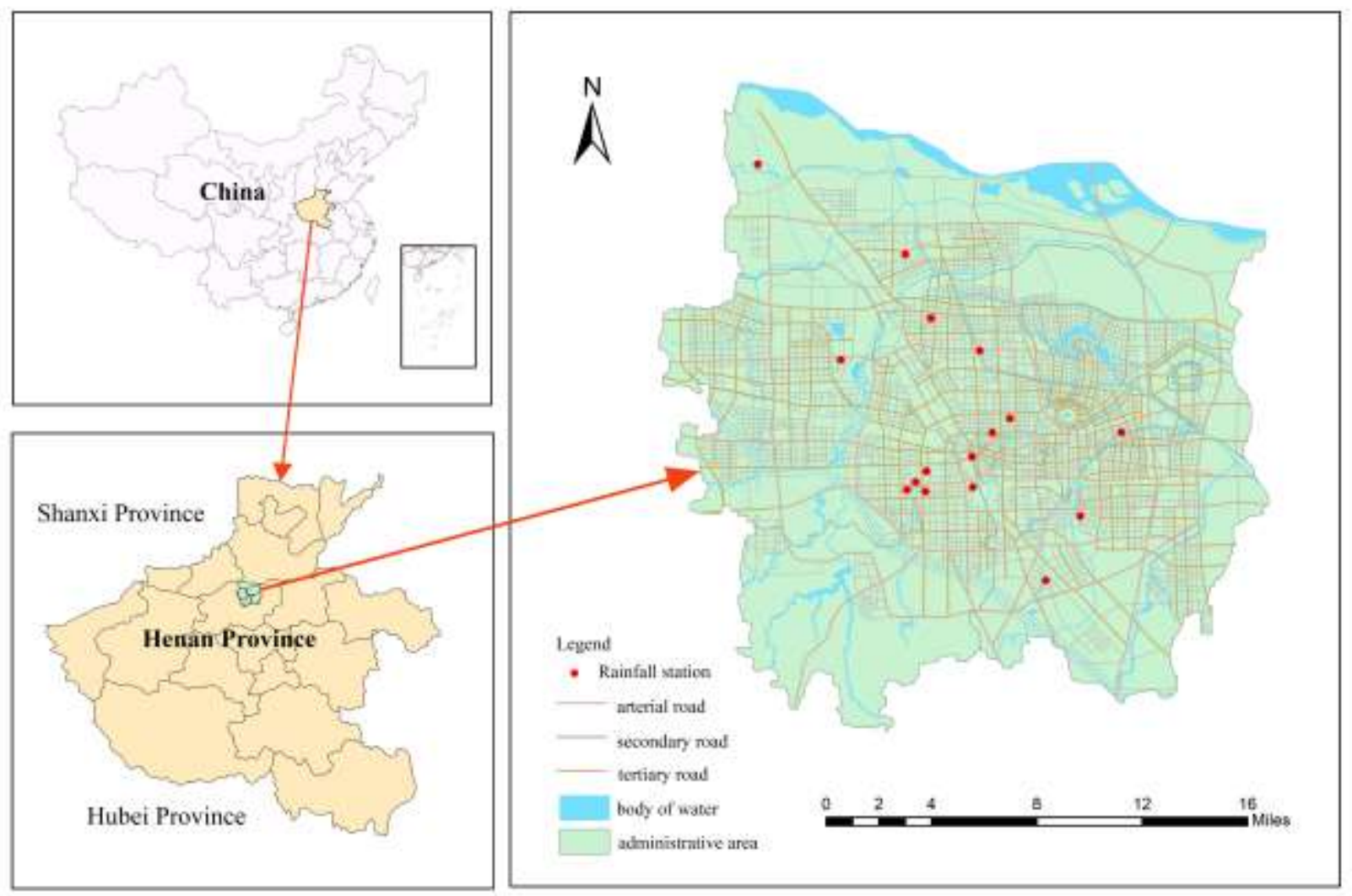

Fig. 1. The Location of study area

\subsection{Data}

\subsubsection{Rainfall observation data}

Rainfall observation data refer to the time-history distribution data of rainfall observed and recorded by 16 rainfall stations, which come from the Zhengzhou City Meteorological Department (Fig. 1). The time resolution of the data is 10 minutes. In this study, 21 historical rainfall data points from 2016 to 2018 were selected as the sample data of the model: June 4, 2016, June 23, 2016, July 19, 2016, August 4, 2016, August 6, 2016, August 25, 2016, September 12, 2016, May 22, 2017, June 5, 2017, June 22, 2017, July 18, 2017, August 12, 2017, August 25, 2017, August 30, 2017, May 15, 2018, July 4, 2018, July 13, 2018, July 27, 2018, August 10, 2018, September 15, 2018, and September 25, 2018. To obtain the rainfall spatial distribution data, the kriging interpolation method was used to perform interpolation analysis on 
the rainfall time-history distribution data of each rainfall station to obtain the rainfall time-history distribution data of each ponding point.

\subsubsection{Ponding observation data}

Ponding observation data refer to the ponding depth of ponding points in historical ponding events. In this study, the ponding depth data of 48 typical ponding points were collected, which were obtained from Zhengzhou Municipal Department. The time resolution of the data was 1 minute. The ponding depth data of these ponding points was from the ponding detection equipment at each road intersection.

\subsubsection{Rainfall forecast data}

The rainfall forecast data in the next two hours were obtained by calling the API of Caiyun technology. The rainfall forecast data have a temporal resolution of 1 minute and a spatial resolution of $1 \mathrm{~km}$. It should be noted that since the update period of the observational rainfall data of the rainfall station is 10 minutes, the update period of rainfall forecast data is also taken as $10 \mathrm{~min}$ to ensure the data comparison consistency. Therefore, in this study, a total of 6 updated rainfall forecast data points on August 1, 2019, with forecast periods of 10 minutes, 20 minutes..., 60 minutes, were used as the sample forecast and early warning data, and each update of the sample data included the rainfall forecast data of the 48 ponding points.

\subsection{Training dataset}

The training and testing process of sample data is the basis of constructing the prediction model of the inundation process. To input the process data of these variables into the model, the data processing method of equal distance splitting and reorganizing was used to process the sample data, which is referred to in previous research (Wu et al., 2020). On this basis, calculate the 
sensitivity index (rainfall, rainfall duration, rainfall peak, position coefficient, rain intensity variance and peak multiplier) of each rainfall process after splitting and reorganizing. The location coefficient is the ratio of the length of the time period from the beginning of rainfall duration to the occurrence of the rainfall peak of the rainfall duration. Rainfall intensity variance is the variance in rainfall intensity, which is a statistical index to describe the rainfall pattern. The peak multiplier is the ratio of the rainfall during the peak period to the total rainfall.

The historical rainfall process data, historical ponding process data and rainfall forecast data were processed by using the above sample data procedural processing method, and 35,424 sample data points were obtained, where the test samples are the rainfall process and ponding process data of three randomly selected rainfall events (with different rainfall intensities and durations), with a total of 2,592 test samples, the training samples are the rainfall process and ponding process data of the remaining 18 rainfall events, with a total of 31,104 training samples, and the rainfall forecast sample data were rainfall events on August 1, 2019, with a total of 1,728 sample data points.

\section{Methodology}

\subsection{Spatial autocorrelation analysis}

Spatial autocorrelation is proposed based on the first law of geography. The first law of geography notes that things distributed in space are interrelated, and the closer the distance between things, the closer the relationship between them (Tobler, 1970). Spatial autocorrelation refers to the relationship between a certain attribute of geographical things distributed in a region and the same attribute of other things (Gatrell, 1989). For example, if an attribute of adjacent objects in space has a similar trend and value, the adjacent objects have a positive spatial 
correlation; in contrast, if an attribute of an adjacent object in space has the opposite trend and value, the adjacent object has a negative spatial correlation.

Rainfall is a phenomenon in which the water vapor in the atmosphere falls to the surface in the form of liquid water after condensation. Therefore, the spatial distribution of rainfall usually has strong spatial autocorrelation. The spatial autocorrelation of the water accumulation point refers to whether the ponding process of each ponding point is independent; if the spatial autocorrelation is high, the ponding process of other ponding points will affect the ponding process of the adjacent water accumulation point. The reason may be that there is a strong hydraulic connection between different water accumulation points. In contrast, the low spatial autocorrelation of the ponding points indicates that the ponding point ponding process is independent, and the ponding process of each ponding point is less affected by the ponding process of the surrounding ponding points. In this paper, the spatial autocorrelation between rainfall and ponding points is analyzed by a rainfall ponding process.

Spatial autocorrelation describes the spatial autocorrelation of statistical objects by analyzing the position and attributes of objects (Overmars et al., 2003). Moran's I index and gear's C ratio are commonly used statistical analysis indices, and Moran's I index is the most intuitive and commonly used spatial autocorrelation analysis method. Therefore, this paper uses Moran's I statistics to analyze the spatial autocorrelation of rainfall and ponding points.

Moran's I index characterizes the spatial distribution relationship of a certain attribute of adjacent objects, with a value of $-1 \sim 1$. A positive value indicates that the spatial distribution of a certain attribute of the adjacent object has a positive spatial correlation, and a negative value indicates that the spatial distribution of a certain attribute of adjacent objects has a negative 
spatial correlation. The closer the value is to 0 , the smaller the spatial autocorrelation of a certain attribute between adjacent objects. A value of zero indicates that there is no spatial correlation. The calculation formula of Moran's I index is as follows:

$$
I=\frac{n_{a} \sum_{i=1}^{n_{s}} \sum_{j=1}^{n_{b}} w_{i j}\left(y_{i}-\bar{y}\right)\left(y_{j}-\bar{y}\right)}{\left(\sum_{i=1}^{n_{n}} \sum_{j=1}^{n_{s}} w_{i j}\right) \sum_{i=1}^{n_{s}}\left(y_{i}-\bar{y}\right)^{2}}
$$

where $n_{a}$ is the sample point or the number of grids, $y_{i}$ or $y_{j}$ represents the attribute value of the $i$ or $\mathrm{j}$ point area, and $\mathrm{w}_{\mathrm{ij}}$ is the weight matrix that measures the relationship between the spatial objects $i$ and $j$.

Moran's I index assumes that the distribution of spatial objects is random and then tests whether the hypothesis is tenable by $\mathrm{Z}$ score. It is generally believed that when the score of its normal statistics ( $\mathrm{Z}$ score) is greater than 1.96 , the original hypothesis is rejected; that is, the spatial objects have a significant positive correlation under $95 \%$ probability. In contrast, when the $\mathrm{Z}$ value is less than 1.96 , the original hypothesis is accepted; that is, the spatial objects have no significant positive correlation at $95 \%$ probability, and the distribution of spatial objects is random.

\subsection{Model construction of ponding process at ponding point}

In this study, the GBDT, SVM and BPNN algorithms are used to build the rainfall process and ponding process relationship model. It should be noted that the prediction model of the ponding process is built for each ponding point. For each ponding point, a ponding process prediction model constructed by the GBDT, SVM and BPNN algorithms is required.

\subsubsection{GBDT}

GBDT is an integrated learning algorithm that combines decision trees and gradient boosting 
algorithms (Friedman, 2001). The core idea of GBDT is iterative learning based on the residuals predicted by the decision tree, and finally, the weak learners of each iteration are accumulated and output. CART was selected as the base learner in the process of GBDT training because the CART decision tree structure is simple, easy to understand, and robust. That is, in each iteration, the gradient boosting algorithm is used to make the latter decision tree train the previous tree residual along the direction of the maximum descending gradient, and finally, the classification results of all trees are accumulated and output (Deng et al., 2019).

The number of weak learners, maximum depth and learning rate are the main parameters of GBDT (Wu et al., 2020). The number of weak learners reflects the number of model iterations, which increases with the increase in the number of iterations. Max depth refers to the maximum depth of the decision tree, which is "none" by default. However, due to the large quantity of data in this study, it is necessary to reasonably set the number of trees to prevent overfitting. The learning rate is a parameter between 0 and 1 that represents the shrinkage step in the update process. The above parameters are optimized by the grid search algorithm. A complete mathematical and technical description of GARP model can be found in Friedman., 2001, Wu et al., 2020.

\subsubsection{SVM}

The SVM algorithm is a supervised machine learning algorithm proposed by Vapnik and Corts in 1995 (Cortes and Vapnik, 1995). It improves the generalization ability of the learning machine by minimizing the empirical risk and structured risk so that when the number of samples is small, a good statistical law can be obtained (Zhou et al., 2020). The core idea of SVM technology is to use kernel function $\mathrm{K}(\mathrm{Xi}, \mathrm{XJ})$ to map the data to a high-dimensional or even infinite dimensional 
feature space so that the data can be easily processed by a linear learning machine in the obtained feature space. On this basis, the optimal hyperplane can be found to solve the problem that the sample data cannot be fitted in the low dimensional space.

SVM can solve both classification and regression problems. In this paper, the regression method is used to predict the ponding process. Assume the training sample $\mathrm{D}=\left\{\left(\mathrm{x}_{1}, \mathrm{y}_{1}\right),\left(\mathrm{x}_{2}, \mathrm{y}_{2}\right)\right.$, $\left.\left(\mathrm{x}_{\mathrm{n}}, \mathrm{y}_{\mathrm{n}}\right)\right\}$, where yi is the observation value of the objective function corresponding to $\mathrm{xi}$, and the ultimate goal of the regression support vector machine is to find the regression fitting function $f(x)=\omega \varphi(x)+b$, where $\varphi(x)$ is the mapping function, which is used to map the sample to a linearly separable high-dimensional space. Set the estimated value of the sample data to not less than $\varepsilon$.

To find the optimal $\omega$ and $\mathrm{b}$, it is transformed into the following optimal solution problem:

SVM provides a variety of kernel functions, such as the linear kernel function, polynomial kernel function, radial basis kernel function and sigmoid kernel function. Among them, the radial basis kernel function is widely used and has higher efficiency for nonlinear data mapping (Xiao et al., 2019). Therefore, the radial basis function was used as the kernel function of the support Nayak and Ghosh, 2013, Wang and Song, 2019.

\subsubsection{BPNN}

A BPNN is a backpropagation neural network connected by multiple neurons, which can be 
divided into an input layer, hidden layer and output layer. BPNN adopts a full interconnection mode between layers, and there is no connection between neurons in the same layer. The BPNN transmission is divided into the forward propagation stage and the backpropagation stage. In the forward propagation stage, the signal starts to propagate from the input layer, and the deviation is calculated in the output layer. If the deviation meets the requirements, the program is terminated. If the deviation does not meet the requirements, the program enters the backpropagation stage. In the backpropagation stage, the weight of each layer is modified by calculating the local gradient of the network and then the forward propagation stage is entered again after the network is reassigned. The program is terminated when the deviation meets the requirements. The grid search algorithm was also used to optimize the main parameters of BPNN (learning rate, number of hidden layers, number of nodes in hidden layers). A complete description of BPNN model can be found in Jiang and Hong, 2013, Li et al., 2019.

\subsection{Model accuracy analysis} of each model attribute and the overall model predictive performance. MAE refers to the average consistency level to a certain extent. CC refers to the correlation degree (Equation 5) between the 
closer the $\mathrm{CC}$ is to 1 , the better the prediction result consistency. NSE is a commonly used parameter to test the simulation quality of the hydrological model (Equation 6). The closer NSE (the value is negative infinity to 1 ) is to 1 , the better the model quality is and the higher the model credibility. Therefore, MAE, RMSE, CC, and NSE were used to evaluate and compare the performance of the models in this study.

where $\mathrm{xi}$ is the predicted value of the sample, yi is the measured value, $\bar{x}$ is the mean value of the predicted sample, $\overline{\mathrm{y}}$ is the mean value of the measured sample, and $\mathrm{n}$ is the total number of samples.

Precision and recall were used to evaluate the performance of early warning results. Precision refers to the proportion of true positive samples in prediction samples (Faceli et al., 2011), which reflects how many prediction results are true (Equation 7). Recall (Equation 8) refers to the proportion of true positive samples in all positive samples (Faceli et al., 2011).

$$
\begin{gathered}
\text { Precision }=\frac{T P}{T P+F P} \\
\text { Recall }=\frac{T P}{T P+F N}
\end{gathered}
$$

where TP is the number of samples correctly classified as positive (Faceli et al., 2011). TN is the 
number of samples correctly classified as negative (Faceli et al., 2011). FP is the number of samples incorrectly classified as positive because the right category is negative (Faceli et al., 2011). FN is the number of samples incorrectly classified as negative because the right class is positive (Faceli et al., 2011).

\section{Results}

\subsection{Spatial autocorrelation results}

The spatial statistical analysis software GeoDa developed by the University of Chicago Spatial Data Center was used to analyze the spatial autocorrelation of rainfall and ponding points in this paper. The spatial autocorrelation results of rainfall are shown in Fig. 2(a). The value of Moran's I index is 0.184 , and its normal statistic $\mathrm{Z}$ value is 4.23 , which is greater than 1.96 . Therefore, it is believed that there is a significant positive spatial correlation of rainfall under 95\% probability, which verifies the spatial autocorrelation of rainfall.

Moran's scatter diagram of ponding is shown in Fig. 2 (b), the value of Moran's I is 0.00077, which is close to 0 , indicating that the spatial distribution of ponding has a low correlation. In addition, the test score ( $\mathrm{Z}$ value) of the normal statistic is 0.8648 , which is less than 1.96 , indicating that the spatial distribution of ponding is irrelevant under $95 \%$ probability. This result shows that there is no obvious hydraulic connection among the ponding points and that they are independent of each other in space. Therefore, it is theoretically feasible to construct a rainfall and ponding process relationship model for each ponding point. 


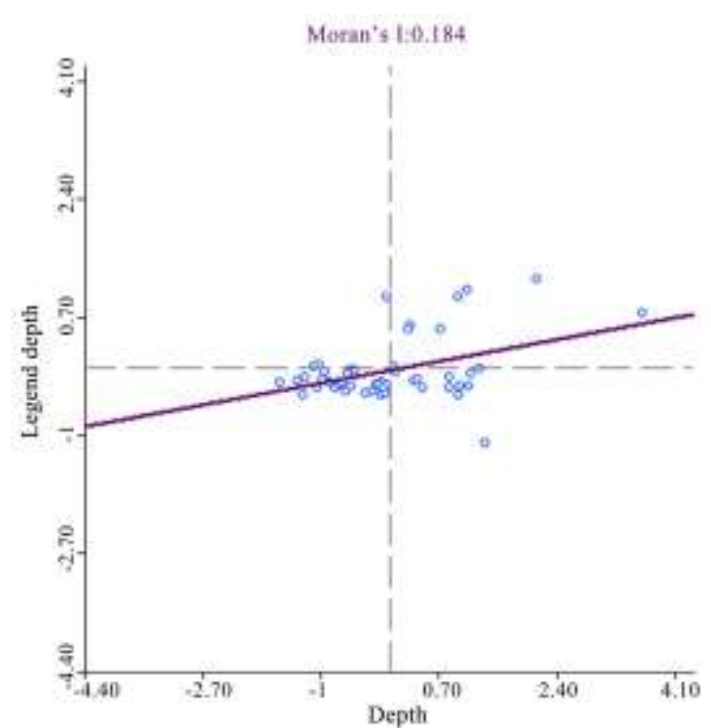

(a) Rain

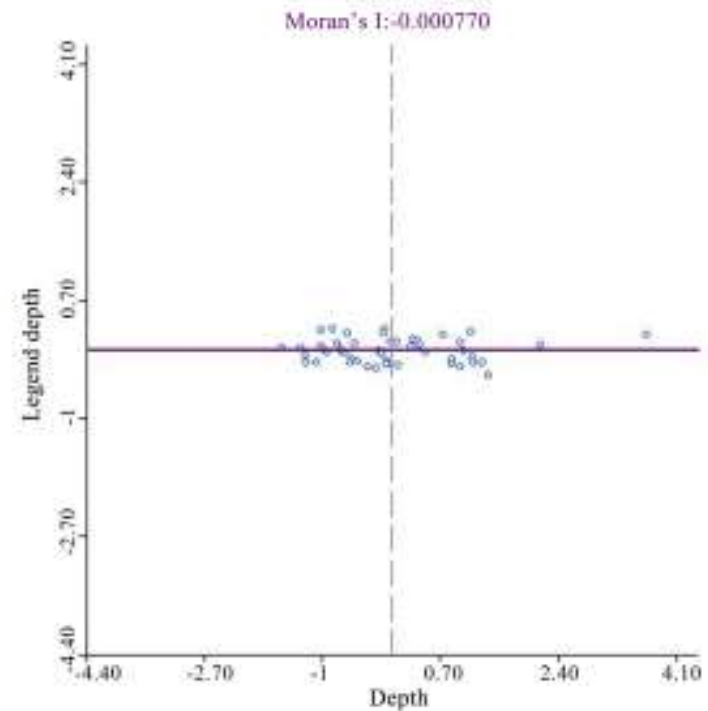

(b) Ponding points

Fig. 2. Moran scatter diagram of rainfall and ponding

\subsection{Analysis and comparison of prediction model results}

In this study, Python 3.7 developed by Google was used to train and verify the model. In the model training process, the 18 randomly selected sample datasets are read into the model in CSV format by using the Python data import module, completing the training of the GBDT, SVM and BPNN models. In the verification phase, the sample datasets of the three randomly selected rainfall events are input into the model as verification data, and MAE, RMSE, CC, and NSE are used to evaluate and compare the performance of the model. As shown in Table 1, the mean absolute error of GBDT, SVM and BPNN for the ponding depth prediction are not greater than $0.03 \mathrm{~m}$, and the $\mathrm{CC}$ between the prediction results and the measured results is greater than 0.97 , which shows the effectiveness of these three algorithms in the ponding depth prediction to a certain extent. However, in comparison, the RMSE of the GBDT prediction model is significantly lower than that of SVM and BPNN, indicating that the GBDT model is more stable and more robust for predicting ponding depth. The stability of the model prediction results is 
often very important for the prediction of urban flood depth. Therefore, from the perspective of the above statistical evaluation indicators, the GBDT prediction model is more suitable for urban flood depth prediction.

Table 1 The prediction performance of GBDT, SVM and BPNN

\begin{tabular}{ccccc}
\hline Category & MAE $(\mathrm{m})$ & RMSE $(\mathrm{m})$ & CC & NSE \\
\hline SVM & 0.0220 & 0.0427 & 0.9878 & 0.9538 \\
BPNN & 0.0204 & 0.0313 & 0.9937 & 0.9776 \\
GBDT & 0.0107 & 0.0207 & 0.9953 & 0.9880 \\
\hline
\end{tabular}

In addition, Fig. 3, Fig. 4 and Fig. 5 show the prediction performance of the SVM, BPNN and GBDT prediction models in different validation events and different ponding points. In the first verification event, the absolute error (AE) range and MAE of the GBDT (0 0.0405 mm and $0.0122 \mathrm{~mm}$ ) prediction model are significantly lower than those of the SVM (with 0.0001 0.0922 mm AE and $0.025 \mathrm{~mm}$ MAE) and BPNN (with 0.0014 0.0973 mm AE and 0.024 mm MAE). Similarly, in the second verification event, the AE range and MAE of the GBDT prediction model are $0 \sim 0.0450 \mathrm{~mm}$ and $0.0090 \mathrm{~mm}$, respectively, which are also lower than those of SVM (with 0.0001 0.0992 $\mathrm{mm} \mathrm{AE}$ and $0.0166 \mathrm{~mm} \mathrm{MAE}$ ) and BPNN (with 0 0.0823 mm AE and $0.0203 \mathrm{~mm}$ MAE). Similarly, the AE range and MAE of the GBDT prediction model (0 0.4667 $\mathrm{mm}$ and $0.0110 \mathrm{~mm}$ ) are lower than those of SVM (with 0.0001 0.0988 $\mathrm{mm} \mathrm{AE}$ and 0.0248 mm MAE) and BPNN (with 0.0001 0.0912 $\mathrm{mm}$ AE and 0.0218 mm MAE). Therefore, it is not difficult to find that the performance of the GBDT prediction model is superior to that of SVM and BPNN in different validation events. At different ponding points, as shown in Fig. 3, Fig. 4 and Fig. 5, the AE range and MAE of the prediction results are quite different. This phenomenon is a common feature of SVM, BPNN and GBDT models. However, the MAE of the 

obviously more stable.

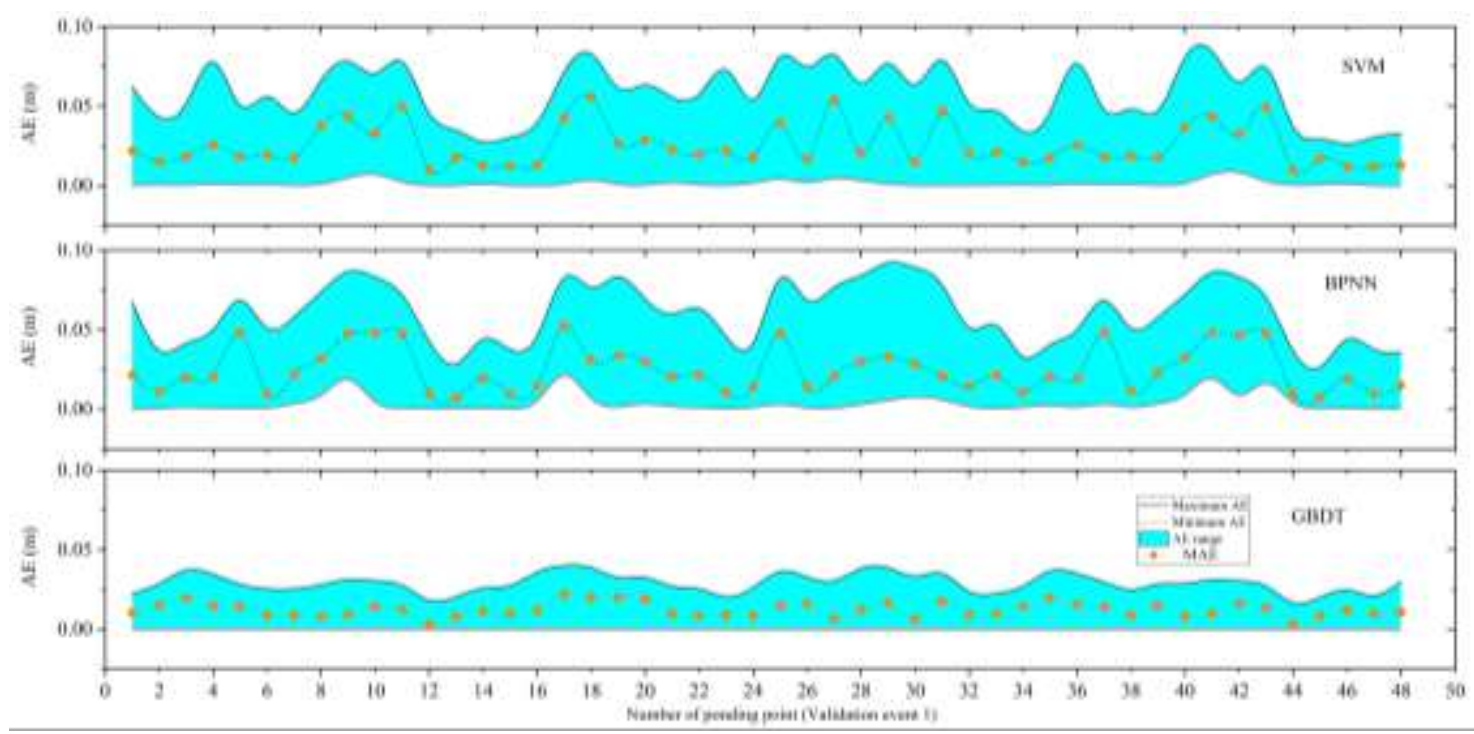

Fig. 3.AE of prediction results of different ponding points in the first verification event

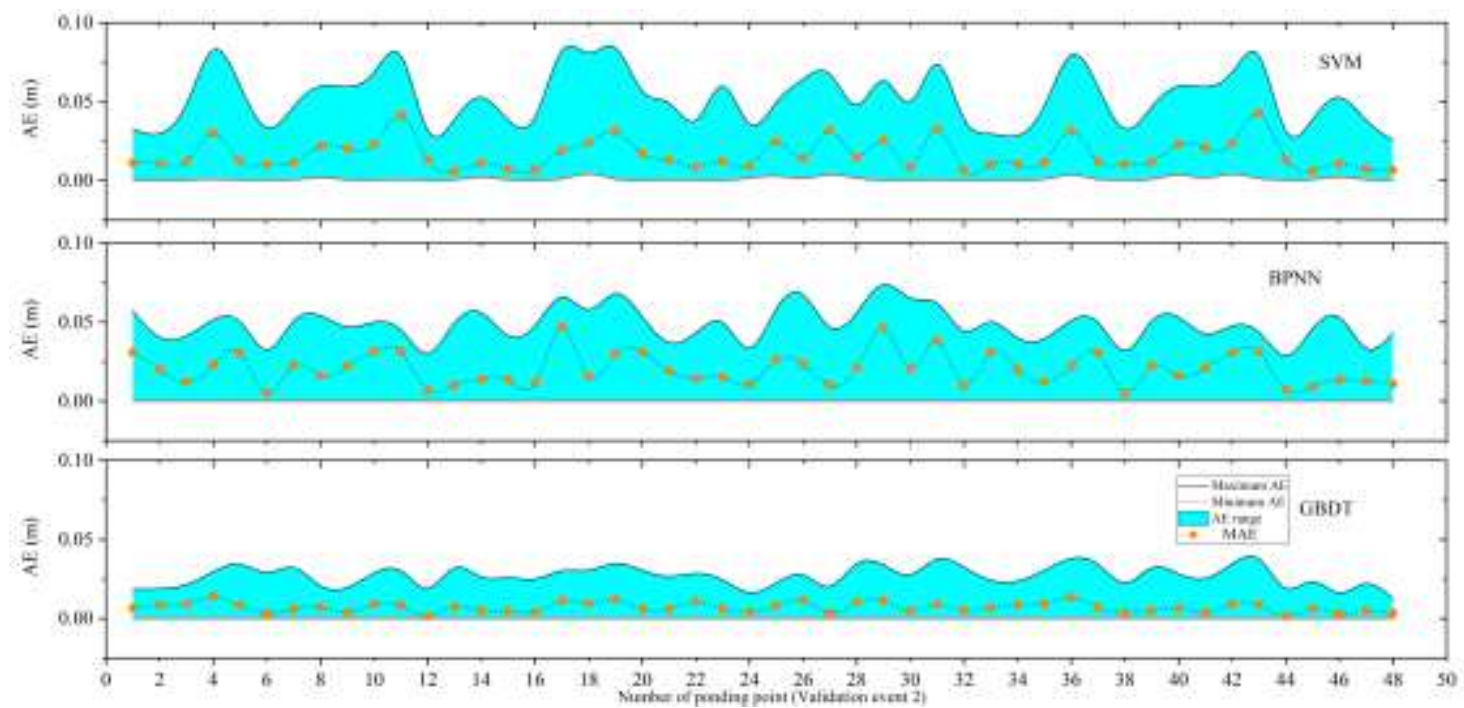

Fig. 4.AE of prediction results of different ponding points in the second verification event 


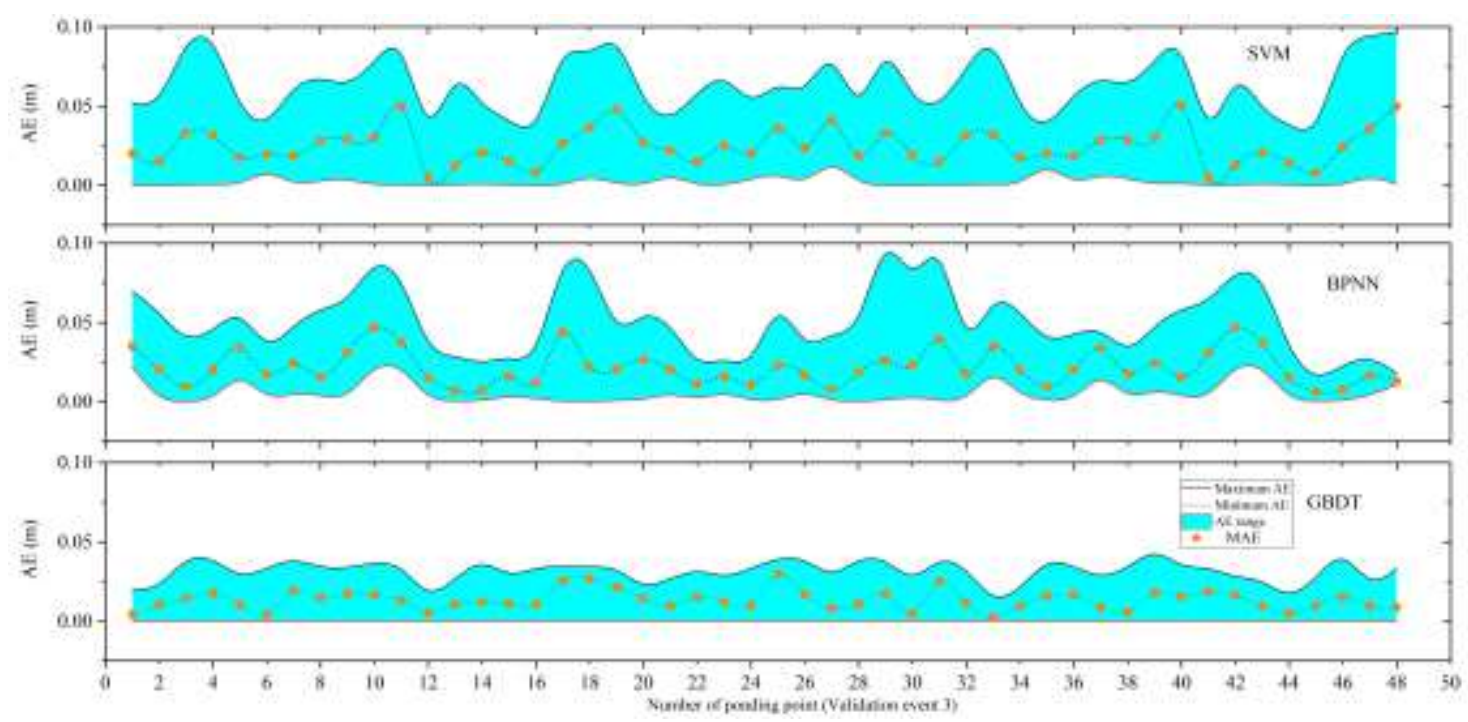

Fig. 5.AE of prediction results of different ponding points in the third verification event

To more clearly compare the difference between GBDT, SVM and random forest in the prediction of ponding depth, the prediction results of the ponding depth of four ponding points (\# $1, \# 5, \# 9, \# 13$ ) in a rainfall event were extracted by a random sampling method. As shown in Fig. 6, the ponding hydrograph at ponding point No. 1 was relatively flat, and the ponding depth fluctuated slightly from 8 to 20 minutes, which was obviously different from the other three ponding points. The reason for this phenomenon may be that the catchment area of ponding point No. 1 is larger than that of the other three ponding points. After rainwater reaches the surface, rainwater near the ponding point quickly collects at the ponding point to form ponds. However, the rainwater far away from the ponding point takes longer to converge to the ponding point after reaching the surface, which causes the ponding point to still have considerable rainwater converging to the ponding point after the ponding reaches the peak value, extending the duration of the ponding peak value. The other three ponding points have similar ponding formation and dissipation processes, and the ponding subsides soon after reaching the maximum depth. It is worth noting that the prediction results of the GBDT model for the ponding peak are very close (with 0.009 MAE) to the measured results, and the prediction accuracy is significantly better 

in predicting the ponding peak.


Fig. 6. Prediction results of ponding process (part)

\subsection{Feature importance analysis}

The information gain ratio (IGR) is an effective feature importance selection method. The larger the IGR of the condition factor is, the higher the influence of the condition factor on the prediction ability of the model. Therefore, in this study, the feature importance of each feature variable (rainfall, rainfall duration, rainfall peak, position coefficient, peak multiplier ratio, and rain intensity variance) on the influence of ponding depth was calculated based on the IGR.

As shown in Fig. 7, the result of feature selection shows that rainfall $(0.2893)$ is the most important factor affecting the ponding depth, and their cumulative contribution is more than $80 \%$. 
The reason is that rainfall is the driving factor of ponding, and only sufficient rainfall will produce ponding. In addition, the rainfall peak value (0.2070) and position coefficient $(0.1864)$ are also important factors affecting the ponding depth. The reason is that the peak rainfall determines the size of the rainfall events and directly affects ponding severity. The position coefficient determines the rainfall pattern characteristics, and different types of rainfall patterns also have different degrees of impact on ponding depth. In contrast, the peak multiple ratio (0.1148) and rainfall intensity variance (0.0511) have little influence on the ponding prediction, indicating that the small rainfall pattern fluctuation has little or even negligible influence on ponding. These results indicate that the ponding depth is more sensitive to rainfall, rainfall peaks and position coefficients. In urban flood forecasting and early warning, we should focus on heavy rainfall events.

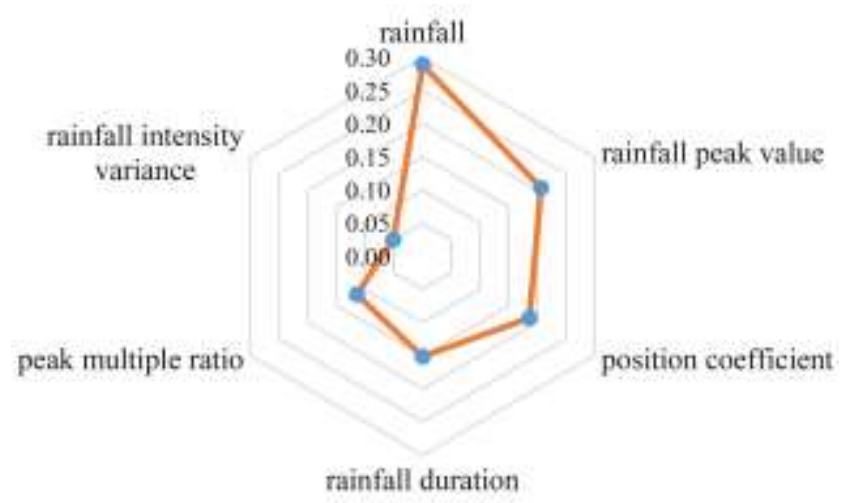

Fig.7. Feature importance analysis results

\subsection{Prediction and early warning of the ponding process driven by rainfall forecast data}

It can be seen in 4.2 that the prediction effect accuracy and stability of the GBDT prediction model are better than SVM and BPNN. Therefore, the GBDT prediction model was used to predict ponding depth in this study. The rainfall forecast data are the basis for ponding depth prediction. In this study, the rainfall event in Zhengzhou city on August 1, 2019, was taken as the 
forecast event. By calling the API of Caiyun technology, the rainfall forecast data of the 48 ponding points in this event were obtained 60 minutes in advance. The ponding process data of 48 ponding points were obtained by inputting the sensitivity index of rainfall prediction data into the GBDT prediction model. On this basis, four ponding points (\# 9, \# 19, \# 29, \# 39) were selected by the equidistant sampling method to draw the ponding process curve. As shown in Fig. pipe network. In the beginning, the rainwater does not exceed the pipeline drainage capacity, so it will not form ponds. However, when the rainwater collection speed exceeds the pipe network ponding point has a slight lag compared with the rainfall hydrograph.

To more intuitively display the ponding depth prediction results of ponding points, the ponding depth and ponding duration early warning classification method (Table 2) is used to classify the ponding depth of ponding points. The reason is that the early warning classification method based on ponding depth and ponding duration can not only directly reflect the ponding depth but also consider the continuous impact of long-term ponding. 

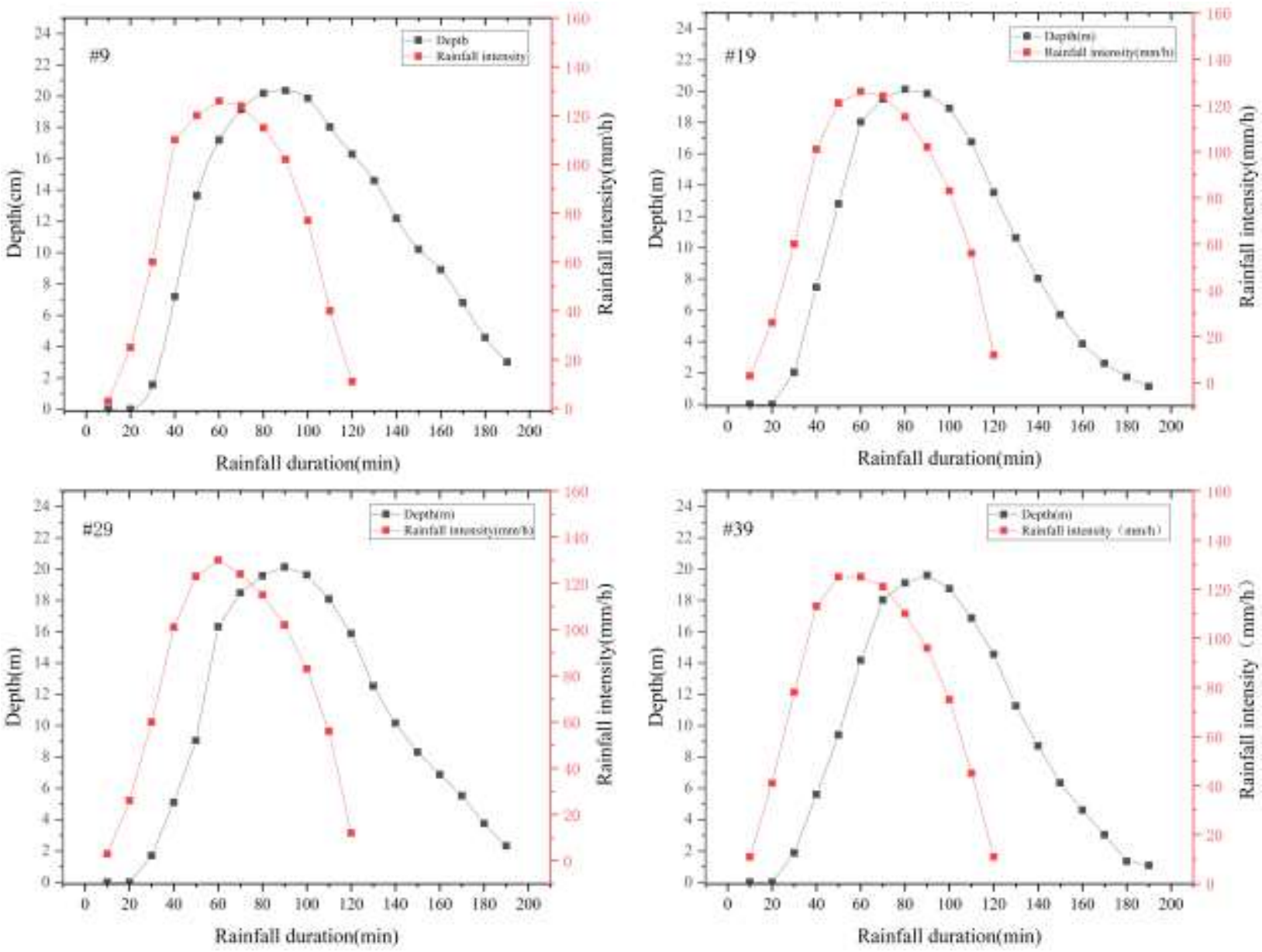

Fig. 8. Prediction results of ponding process at ponding points under the prediction period of

$60 \mathrm{~min}$ in advance (part)

Table 2 Classification standard of ponding early warning level

\begin{tabular}{cccc}
\hline Early warning level & Ponding depth $(\mathrm{cm})$ & Ponding duration (min) & The degree of ponding \\
\hline 1 & $0 \sim 3$ & $/$ & No ponding \\
2 & $3 \sim 10$ & $>10$ & Mild ponding \\
3 & $10 \sim 25$ & $>3$ & Moderate ponding \\
4 & $>25$ & $/$ & Severe ponding \\
\hline
\end{tabular}

By inputting the rainfall forecast data of $60 \mathrm{~min}, 50 \mathrm{~min}, 40 \mathrm{~min} . . ., 10 \mathrm{~min}$ before rainfall into the model, the ponding process data of 48 ponding points at $60 \mathrm{~min}, 50 \mathrm{~min}, 40 \mathrm{~min} . ., 10$ min before rainfall are obtained. Based on this, the maximum depth and ponding duration are selected as early warning indicators, and the spatial processing and display function of GIS are 
used as early warning technology to draw the real-time correction of ponding point early warning results. As shown in Fig. 9, the areas with serious ponding are mainly concentrated in the central and eastern parts of Zhengzhou city, especially in Longhai Road, Hanghai Road and the old urban area in the central area. The reason is that there are many underpass tunnels on Haihang Road and Longhai Road. These underpass tunnels often have the characteristics of low elevation, large terrain slope and wide catchment area. After rainwater reaches the surface, it quickly collects and flows to form ponds at the bottom of the tunnel. Therefore, ponding points with serious ponding mostly appear near these underpass tunnels. In contrast, the degree of ponding in the northern part of Zhengzhou city is obviously lower. The reason may be that the northern part of Zhengzhou is adjacent to the Yellow River wetland, which has a relatively flat terrain and a large proportion of the surface permeable area. Therefore, the amount of infiltration and interception of rainwater after reaching the ground is large, resulting in low confluence and confluence velocities.

To further verify the effectiveness of real-time early warning results of ponding points using rainfall forecast data, the ponding depth of each rainfall ponding point on August 1, 2019, was obtained by means of ponding monitoring equipment, electronic water gauges and actual measurements, and the early warning results and measured results of ponding points were analyzed by the accuracy evaluation index of precision and recall. As shown in Table 3, the overall precision and recall of real-time ponding point early accuracy results is more than $80 \%$, indicating that the overall early warning result precision can meet the requirements of urban flood forecasting and early warning. Moreover, the prediction precision shows an overall trend of improvement, although the prediction precision fluctuates with the shortening of the forecast 
period. For example, the prediction precision in the next 10 minutes is $14.7 \%$ higher than that in

429 the next 60 minutes. Moreover, from the perspective of the local accuracy of the early warning

430 results, the prediction precision of ponding level 4 (i.e., serious ponding) is obviously higher than

431 that of other levels in the early warning results of different ponding point levels (Table 3), which

432 indicates that the GBDT algorithm has obvious advantages for predicting more serious ponding.

Table 3 Statistics of early warning results of ponding points

\begin{tabular}{|c|c|c|c|c|c|c|c|c|c|c|}
\hline \multirow{2}{*}{$\begin{array}{c}\text { Ponding } \\
\text { level }\end{array}$} & \multicolumn{2}{|c|}{1} & \multicolumn{2}{|c|}{2} & \multicolumn{2}{|c|}{3} & \multicolumn{2}{|c|}{4} & \multirow{2}{*}{$\begin{array}{c}\text { Precision } \\
(\%)\end{array}$} & \multirow{2}{*}{$\begin{array}{c}\text { Recall } \\
(\%)\end{array}$} \\
\hline & $\mathrm{TP}$ & $\mathrm{FP}$ & $\mathrm{TP}$ & $\mathrm{FP}$ & $\mathrm{TP}$ & $\mathrm{FP}$ & $\mathrm{TP}$ & FP & & \\
\hline Measured & \multicolumn{2}{|c|}{5} & \multicolumn{2}{|c|}{13} & \multicolumn{2}{|c|}{19} & \multicolumn{2}{|c|}{11} & & \\
\hline 10min early & 5 & 0 & 12 & 0 & 19 & 0 & 11 & 1 & 97.9 & 98.1 \\
\hline 20 min early & 5 & 1 & 10 & 1 & 19 & 1 & 10 & 1 & 90.0 & 92.0 \\
\hline 30 min early & 5 & 1 & 10 & 2 & 17 & 3 & 10 & 0 & 87.9 & 89.3 \\
\hline 40 min early & 5 & 1 & 10 & 2 & 17 & 3 & 9 & 1 & 85.4 & 87.1 \\
\hline 50 min early & 4 & 1 & 9 & 1 & 19 & 2 & 10 & 2 & 86.0 & 85.0 \\
\hline 60min early & 4 & 1 & 11 & 3 & 16 & 3 & 9 & 1 & 83.2 & 82.7 \\
\hline Precision (\%) & \multicolumn{2}{|c|}{85.0} & \multicolumn{2}{|c|}{87.7} & \multicolumn{2}{|c|}{89.9} & \multicolumn{2}{|c|}{90.9} & & \\
\hline
\end{tabular}



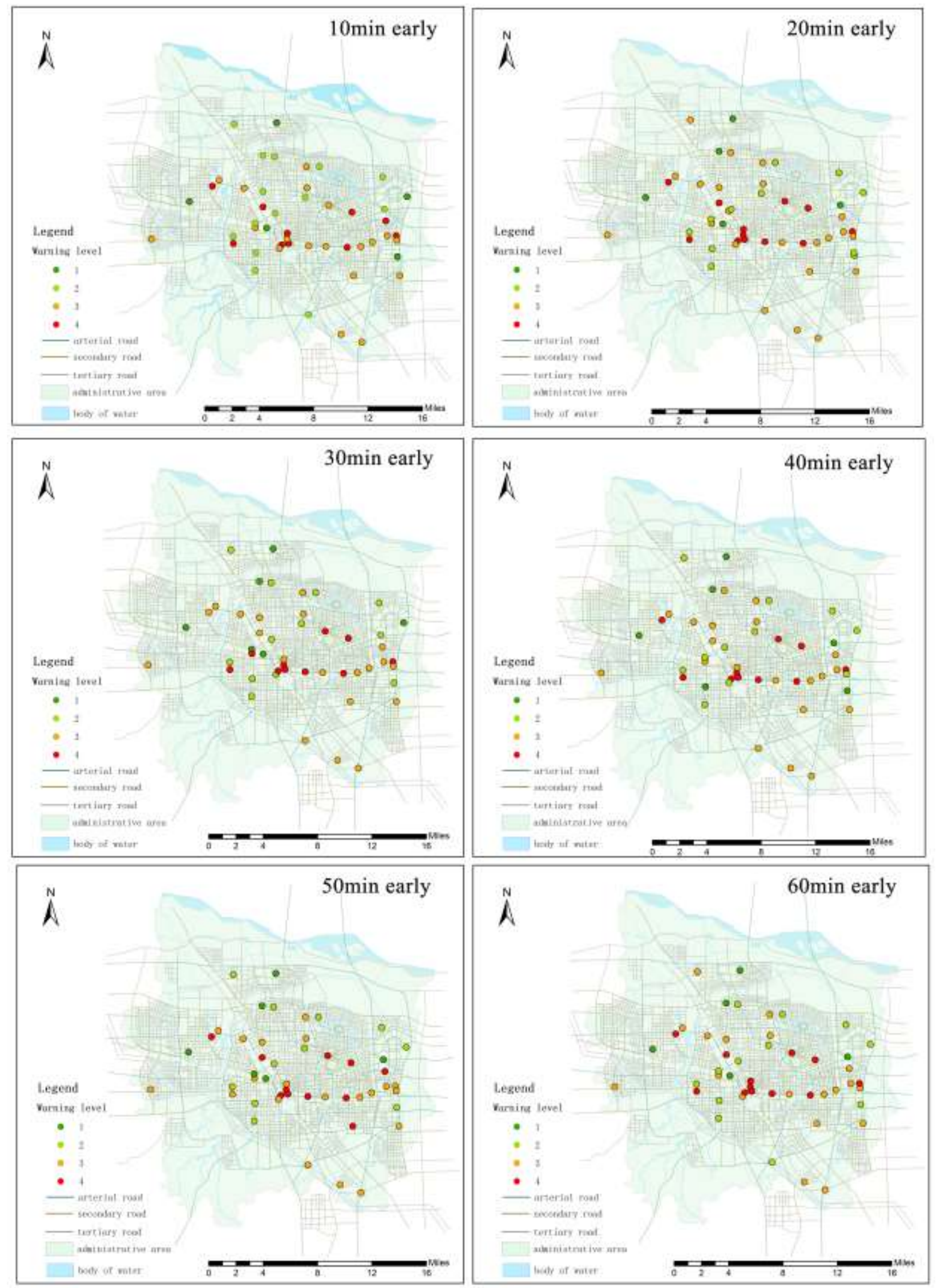

Fig. 9. Early warning level map of ponding point (10 60min early) 


\section{Discussion}

As seen in Fig. 2 and Fig. 9, although the ponding points do not have spatial correlation, the ponding points with the same ponding grade still have certain spatial aggregation. Specifically, ponding in the central region is more serious, which is mainly due to the combined influence of the spatial correlation of rainfall (Wang et al., 2020), the urban heat island effect and the distribution (Min et al., 2018) of urban functional areas. The urban heat island effect makes the rainfall in the center of the city larger. The city center is a densely populated old city with lower pipe network design standards, aging and severely damaged pipe networks and higher impervious areas. After rainwater reaches the surface, there is less infiltration, faster water collection speed, and limited drainage capacity, which easily causes more serious ponding in the central part of the city. In addition, Fig. 7 shows that urban ponding is most sensitive to rainfall, rainfall peaks and location coefficients. Therefore, to reduce losses caused by floods, urban management departments should not only increase the area of permeable ground in the central region of urban areas and transform, repair and dredge the drainage pipe network but also take timely measures such as drainage and cutting off roads when dealing with heavy rainfall.

In terms of the early warning results accuracy, as shown in Table 3, with the shortening of the forecast period, the prediction accuracy shows an overall improvement trend. The main reason is that rainfall forecast data accuracy gradually improves, and the accuracy of input variables improves with the shortening of the forecast period, which leads to the improvement of prediction variable accuracy. It is generally believed that there is a contradiction between the forecast period and the prediction accuracy. The shortening of the forecast period will result in higher prediction accuracy, and a longer forecast period will reduce the prediction accuracy. 
Fortunately, the prediction precision and recall of the model proposed in this study still exceed $80 \%$ when the forecast period is 60 minutes. It effectively guarantees a certain forecast accuracy while obtaining a longer forecast period, which effectively addresses one of the contradictions between the forecast period and the prediction accuracy. The research results can provide more guiding theoretical and technical references for improving prediction and early warning methods and preventing flood disasters.

\section{Conclusion}

In this study, constructing ponding process prediction and early warning methods for ponding points is systematically explained in three aspects: method feasibility, method comparison and screening, and practical application of methods. The conclusions are as follows:

(1) The feasibility of this study is explained by using the spatial autocorrelation analysis method, that is, each ponding point is spatially independent of each other, and there is no spatial correlation. Therefore, the urban flood inundation process model based on ponding points is theoretically feasible.

(2) Based on different deep learning methods (GBDT, SVM, and BPNN), the relationship model between the rainfall process and the ponding process is constructed for each ponding point. A statistical evaluation method was used to analyze the applicability of different deep learning methods in ponding depth prediction. The results show that the GBDT algorithm has the highest accuracy for ponding depth prediction, which indicates that it is the most suitable method for the prediction of ponding depth.

(3) Taking the rainfall event in Zhengzhou on August 1, 2019, as an example, a refined realtime prediction model for the urban flood ponding process driven by rainfall forecast data was 
constructed. The real-time early warning for ponding points is realized by the warning level standard and GIS. The results show that the overall accuracy of early warning results is more than $80 \%$, and the accuracy of early warning results shows an upward trend with the shortening of the prediction period, which can meet the urban flood control requirements.

However, due to limitations in rainfall and ponding data, the ponding process prediction model can only be built for ponding points with detailed data in this study. With the gradual enrichment of data and advancement of physical simulation technology, future research can attempt to expand the research scope combined with physical models.

Authors contributions Yihong Zhou: Methodology, Validation, Writing - original draft, Writing - review \& editing. Zening Wu: Writing - review \& editing, Funding acquisition. Mengmeng Jiang: Validation. Hongshi Xu: Methodology. Denghua Yan: Supervision. Huiliang Wang: Methodology, Funding acquisition. Chentao He and Xiangyang Zhang: Data curation.

Funding The work was funded by the Key Project of National Natural Science Foundation of China (No: 51739009), Excellent Youth Fund of Henan Province of China (212300410088), Science and Technology Innovation Talents Project of Henan Education Department of China (1HASTIT011) and Young backbone Teachers Training Fund of Henan Education Department of China (2020GGJS005).

Availability of Data and Materials Data can be made available through contacting the corresponding author.

\section{Declarations}

Ethical Approval Not applicable.

Consent to Participate The authors agree to participate in the journal. 
Consent to Publish The authors providing consent publication of the manuscript to the journal publisher.

Competing Interests The authors declare that they have no known competing financial interests or personal relationships that could have appeared to influence the work reported in this paper.

\section{References}

Babaei S, Ghazavi R, Erfanian M (2018) Urban flood simulation and prioritization of critical urban sub-catchments using swmm model and promethee ii approach. Phys Chem Earth 105:3-11. https://doi.org/10.1016/j.pce.2018.02.002

Bermudez M, Ntegeka V, Wolfs V, Willens P (2018) Development and comparison of two fast surrogate models for urban pluvial flood simulations. Water Resour Manag 32(8):1-15. https://doi.org/10.1007/s11269-018-1959-8

Carreau J, Guinot V (2020) A pca spatial pattern based artificial neural network downscaling model for urban flood hazard assessment - sciencedirect. Adv Water Resour 147:103821. https://doi.org/10.1016/j.advwatres.2020.103821

Cortes C, Vapnik V (1995) Support-vector networks. Mach Learn 20:273-297. https://doi.org/10.1023/A:1022627411411

Deng SK, Wang CG, Wang MY, Sun Z (2019) A gradient boosting decision tree approach for insider trading identification: An empirical model evaluation of China stock market. Appl Soft Comput 83:105652. https://doi.org/10.1016/j.asoc.2019.105652

Faceli K, Lorena AC, Gama JA, Carvalho A et al (2011) Inteligência Artificial: Uma abordagem de aprendizado de máquina. 2:192

Friedman JH (2001) Greedy Function Approximation: A Gradient Boosting Machine. Ann Stat 
Gatrell AC (1989) Book reviews: Griffith, D.A. 1987: Spatial autocorrelation: a primer. Pennsylvania: Association of American Geographers. iv + 82 pp. US \$6.00. Prog. Hum Geog 13:144-145. https://doi.org/10.1177/030913258901300115

Hou JM, Wang N, Guo KH, Li DL, Jing HX, Wang T, Hinkelmann R (2020) Effects of the temporal resolution of storm data on numerical simulations of urban flood inundation. $\mathbf{J}$ Hydrol 589:125100. https://doi.org/10.1016/j.jhydrol.2020.125100

IPCC (2014) In: Core Writing Team, Pachauri, R.K., Meyer, L.A. (Eds.), Climate Change 2014: Synthesis Report. Contribution of Working Groups I, II and III to the Fifth As assessment Report of the Intergovernmental Panel on Climate Change. IPCC, Geneva, Switzerland (151 pp.)

Jiang H, Hong L (2013) Application of bp neural network to short-term-ahead generating power forecasting for pv system. Adv Mater Res 609:128-131. https://doi.org/10.4028/www.scientific.net/AMR.608-609.128

Ke Q, Tian X, Bricker J, Tian Z, Guan GH, Cai HY, Huang XX, Yang HL, Liu JG (2020) Urban pluvial flooding prediction by machine learning approaches - a case study of Shenzhen city, China. Adv Water Resour 145:103719. https://doi.org/10.1016/j.advwatres.2020.103719

Kenney MA, Janetos AC (2020) National indicators of climate changes, impacts, and vulnerability. Climatic Change 163:1695-1704. https://doi.org/10.1007/s10584-020-02939-4

Lei XX, Chen W, Panahi M, Falah F, Rahmati, Uuemaa E, Kalantari Z, Ferreira CSS, Rezaie F, Tiefenbacher JP, Lee S, Bian HY (2021) Urban flood modeling using deep-learning approaches in Seoul, South Korea. J Hydrol 601:126684. 
Leong WC, Kelani RO, Ahmad Z (2019) Prediction of air pollution index (API) using support vector machine

(SVM).

J Environ

Eng

8:103208. https://doi.org/10.1016/j.jece.2019.103208

Li X, Cheng X, Wu W, Wang Q, Li Y (2019) Forecasting of bioaerosol concentration by a back propagation neural network model. Sci Total Environ 698:134315. https://doi.org/10.1016/j.scitotenv.2019.134315

Ma J, Zhang YF, Li YM, Zhou L, Qin LY, Zeng YW, Wang P, Lei Y (2021) Deep dual-side learning ensemble model for parkinson speech recognition. Biomed Signal Proces 69:102849. https://doi.org/10.1016/j.bspc.2021.102849

Masson V, Lemonsu A, Hidalgo J, Voogt JA (2020) Urban climates and climate change. Annu. Rev Env Resour 45:411-444. https://doi.org/10.1146/annurev-environ-012320-083623

Mignot E, Li XF, Dewals B (2018) Experimental modelling of urban flooding: a review. J Hydrol 568:334-342. https://doi.org/10.1016/j.jhydrol.2018.11.001

Min M, Hongbo Z, Changhong M (2018) Spatio-temporal evolution analysis of the urban heat island: a case study of Zhengzhou city, China. Sustainability 10(6):1992. https://doi.org/10.3390/su10061992

Moghadas M, Asadzadeh A, Vafeidis A, Fekete A, Ktter T (2019) A multi-criteria approach for assessing urban flood resilience in tehran, iran. Int J Disast Risk Re 35:101069. https://doi.org/10.1016/j.ijdrr.2019.101069

Mosavi A, Ozturk P, Chau KW (2019) Flood prediction using machine learning models: literature review. Statistics 10:1536. https://doi.org/10.3390/w10111536 
Nayak MA, Ghosh S (2013) Prediction of extreme rainfall event using weather pattern recognition and support vector machine classifier. Theor Appl Climatol 114:583-603. https://doi.org/10.1007/s00704-013-0867-3

Ntelekos AA, Oppenheimer M, Smith JA, Miller AJ (2010) Urbanization, climate change and flood policy in the United States. Climatic Change 103:597-616. https://doi.org/10.1007/s10584-009-9789-6

Overmars KP, Koning GHJD, Veldkamp A (2003) Spatial autocorrelation in multi-scale land use models. Ecol Model 164: 257-270. https://doi.org/10.1016/S0304-3800(03)00070-X

Panahi M, Jaafari A, Shirzadi A, Shahabi H, Rahmati O, Omidvar E, Lee S, Bui DT (2020) Deep learning neural networks for spatially explicit prediction of flash flood probability. Geosci. Front 12:376-389. https://doi.org/10.1016/j.gsf.2020.09.007

Rahebeh A, Romulus C, Hossein SM, Quoc Bao P (2020) Flash-flood susceptibility mapping based on XGBoost, Random Forest and Boosted Regression Trees. Geocarto Int 1-18. https://doi.org/10.1080/10106049.2021.1920636

Schmitt TG, Thomas M, Ettrich N (2004) Analysis and modeling of flooding in urban drainage systems. J Hydrol 299:3-4. https://doi.org/10.1016/j.jhydrol.2004.08.012

Schreider SYu, Smith DI, Jakeman AJ (2000) Climate change impacts on urban flooding. Climatic Change 47:91-115. https://doi.org/10.1023/A:1005621523177

Sharifi A (2020) Trade-offs and conflicts between urban climate change mitigation and adaptation measures: a literature review. J Clean Prod 276:122813. https://doi.org/10.1016/j.jclepro.2020.122813

Singh KK, Pal M, Singh VP (2010) Estimation of mean annual flood in indian catchments using 
backpropagation neural network and m5 model tree. Water Resour Manag 24(10):20072019. https://doi.org/10.1007/s11269-009-9535-x

Sun JY, Shen H, Qu Q, Sun W, Kong XQ (2021) The application of deep learning in electrocardiogram: where we came from and where we should go? Int J Cardiol 337:71-78. https://doi.org/10.1016/j.ijcard.2021.05.017

Suwit O, Parinda P (2016) Urban flood mitigation and prevention using the MIKE 21 model: a case study of Nakhon Ratchasima province, Thailand. Suranaree J Sci Technol 23:461-479. https://doi.org/10.1080/10106049.2021.1920636

Thanvisitthpon N, Shrestha S, Pal I, Ninsawat S, Chaowiwat W (2020) Assessment of flood adaptive capacity of urban areas in thailand. Environ Impact Asses 81:106363. https://doi.org/10.1016/j.eiar.2019.106363

Tobler WR (1970) A Computer Movie Simulating Urban Growth in the Detroit Region. Econ Geogr 46:234-240. https://doi.org/10.2307/143141

Wang B, Loo B, Zhen F, Xi G (2020) urban resilience from the lens of social media data: responses to urban flooding in nanjing, china. Cities 106:102884. https://doi.org/10.1016/j.cities.2020.102884

Wang H, Song L (2019) Water level prediction of rainwater pipe network using an svm-based machine learning method. Int J Pattern Recogn 34:2551002. https://doi.org/10.1142/S0218001420510027

Wang J, Hu CH, Ma B, Mu X (2020) Rapid urbanization impact on the hydrological processes in Zhengzhou, China. Water 12(7):1870. https://doi.org/10.3390/w12071870

Wu ZN, Zhou YH, Wang HY (2020) Real-time prediction of the water accumulation process of 

https://doi.org/10.1109/ACCESS.2020.3017277

615 Xiao JK, Zhu XH, Huang CX, Yang XG, Wen FH, Zhong MR (2019) A new approach for stock 616 price analysis and prediction based on ssa and svm. Int $\mathbf{J}$ Inf Tech Decis 18:87-310. 617 https://doi.org/10.1142/S021962201841002X

$618 \mathrm{Xu} \mathrm{H}, \mathrm{Ma} \mathrm{C}$, Lian J, Xu K, Evance C (2018) Urban flooding risk assessment based on an 619 integrated K-Means cluster algorithm and improved entropy weight method in the region of Haikou, China. J Hydrol 563:975-986. https://doi.org/10.1016/j.jhydrol.2018.06.060 based on PSO-SVM. Socio-Econ Plan Sci 77:101001. https://doi.org/10.1016/j.seps.2020.101001 


\section{Highlights}

- Proposes a prediction method for urban flood process based on different deep learning methods.

- It is feasible to construct prediction model for each water accumulation point.

- Gradient Boosting Decision Tree is the most suitable method for ponding process prediction.

- Rainfall, rainfall peak and location coefficient have the greatest impact on ponding.

- Realize the real-time prediction of urban flood driven by rainfall forecast data. 


\section{Supplementary Files}

This is a list of supplementary files associated with this preprint. Click to download.

- Highlights.doc 